\title{
Abordagem diagnóstica e tratamento da colecistite aguda: uma revisão narrativa
}

\author{
Diagnostic approach and treatment of acute cholecystitis: a narrative review
}

Enfoque diagnóstico y tratamiento de la colecistitis aguda: una revisión narrativa

Emerson Schindler Junior ${ }^{1 *}$, Matheus Henrique Gonzatti ${ }^{1}$, Giórgia Souza Franco ${ }^{1}$, Giovana Rezende Fernandes Costa ${ }^{1}$, Alexia Duarte ${ }^{2}$, Willian Travain ${ }^{3}$.

\section{RESUMO}

Objetivo: Realizar uma revisão da literatura abordando os principais aspectos atuais do diagnóstico e tratamento da colecistite aguda. Revisão bibliográfica: O sinal clínico mais comum é a dor abdominal em hipocôndrio direito ou epigástrio (72-93\%). A ultrassonografia deve ser o primeiro exame solicitado para pacientes com suspeita de colecistite aguda. Outros exames complementares como colangiopancreatografia retrógrada endoscópica, colangiografia intraoperatória ou ultrassonografia endoscópica podem ser necessários para pacientes com médio e alto risco para coledocolitíase. O tratamento da colecistite aguda é cirúrgico, devendo ser realizado da forma mais precoce possível, após serem realizadas as medidas iniciais. A via preferível é a videolaparoscópica. Considerações finais: Uma identificação precoce do quadro de colecistite aguda é fundamental, visto que permite uma abordagem cirúrgica menos complexa, com menores índices de complicação. É indispensável ao médico o conhecimento da apresentação clínica da colecistite aguda, bem como dos métodos de investigação laboratoriais e de imagem, tendo em vista sua significativa incidência.

Palavras-Chave: Colecistite aguda, Colelitíase, Vesícula biliar, Dor abdominal, Videolaparoscopia.

\begin{abstract}
Objective: To perform a literature review about the diagnosis and treatment of acute cholecystitis. Bibliographic review: The main symptom of acute cholecystitis is abdominal pain in the right hypochondrium or epigastrium (72-93\%). Ultrasonography must be the first exam to be requested by any doctor suspecting acute cholecystitis. Further exams, such as endoscopic retrograde cholangiopancreatography, intraoperative cholangiography, or endoscopic ultrasound, may be necessary when the patient has a moderate or high risk of choledocholithiasis. The treatment of acute cholecystitis is surgical, and it should be done as soon as possible, after the initial measures have been taken. The laparoscopic method is the best option. Final considerations: An early diagnosis of acute cholecystitis is important, since it reduces surgical complexity and avoids complications. Due to the significant incidence, doctors must know clinical presentation of acute cholecystitis and its investigation methods such as laboratorial and image.
\end{abstract}

Keywords: Acute cholecystitis, Cholelithiasis, Gallbladder, Abdominal pain, Videolaparoscopy.

\section{RESUMEN}

Objetivo: Realizar una revisión de la literatura sobre el diagnóstico y tratamiento de la colecistitis aguda. Revisión bibliográfica: El síntoma principal de la colecistitis aguda es el dolor abdominal en el hipocondrio

${ }^{1}$ Universidade Positivo (UP), Curitiba - PR. *E-mail: emersonschindlerjr@gmail.com

2 Universidade Federal do Paraná (UFPR), Curitiba - PR.

${ }^{3}$ Hospital Universitário Cajuru (HUC), Curitiba - PR. 
o epigastrio derecho (72-93\%). La ecografía debe ser el primer examen solicitado por cualquier médico que sospeche de colecistitis aguda. Pueden ser necesarios exámenes adicionales, como la colangiopancreatografía retrógrada endoscópica, la colangiografía intraoperatoria o la ecografía endoscópica, cuando el paciente tiene un riesgo moderado o alto de coledocolitiasis. El tratamiento de la colecistitis aguda es quirúrgico y debe realizarse lo antes posible después de las medidas iniciales. El método laparoscópico es la mejor opción. Consideraciones finales: El diagnóstico precoz de colecistitis aguda es importante, ya que reduce la complejidad quirúrgica y evita complicaciones. Debido a su importante incidencia, los médicos deben conocer la presentación clínica de la colecistitis aguda y sus métodos de investigación como las pruebas de laboratorio e imágenes.

Palabras clave: Colecistitis aguda, Colelitiasis, Vesícula biliar, Dolor abdominal, Videolaparoscopia.

\section{INTRODUÇÃO}

A colecistite aguda compreende a doença inflamatória aguda da vesícula biliar, podendo variar de uma condição autolimitada a uma doença potencialmente fatal (CAMPANILLE FC, et al., 2014; ANSALONI L, et al., 2016). O principal fator etiológico da colecistite é a colelitíase, ou seja, a presença de cálculos na vesícula biliar. Em menor proporção pode ser decorrente de: isquemia, distúrbios de motilidade, lesões químicas diretas, infecções, doenças do colágeno e reação alérgica (ITOI T, et al., 2017; KIMURA Y, et al., 2013).

A fisiopatologia da colelitíase aguda envolve a obstrução completa e persistente do colo da vesícula ou do ducto cístico por um cálculo biliar, que leva à estase da bile e consequente irritação química da parede da vesícula. Caso o tratamento não seja instituído, a doença se torna mais grave, podendo resultar em complicações. A evolução patológica compreende a colecistite edematosa, necrosante e supurativa (KIMURA Y, et al., 2013).

Outras formas de colecistite aguda compreendem a colecistite acalculosa, xantogranulomatosa, enfisematosa, gangrenosa e por torção da vesícula biliar. A colecistite acalculosa é característica de pacientes graves, havendo desenvolvimento da doença mesmo na ausência de cálculos biliares. Colecistite xantogranulomatosa ocorre pela infiltração de bile na parede vesicular, causando degradação e formação de granulomas. Na colecistite enfisematosa há formação gasosa na parede e/ou na luz da vesícula biliar oriundo do metabolismo de microorganismos, sendo frequentemente encontrado em idosos diabéticos (KIMURA Y, et al., 2013).

Colecistite gangrenosa ocorre quando a progressão da inflamação e infecção leva ao comprometimento do suprimento arterial da parede da vesícula, levando à isquemia, posterior necrose e com isso, perfuração (GOMES CA, et al., 2019). A colecistite decorrente de uma torção biliar deve ser lembrada quando os achados clínicos e de imagem revelam uma colecistite complicada (ABADÍA-BARNÓ P, et al., 2017).

Os principais fatores de risco relacionados à colecistite são: colelitíase, obesidade, diuréticos tiazídicos, quimioterapia arterial hepática transcateter, terapia de reposição hormonal e AIDS (KIMURA Y, et al., 2013). Já nos casos de colecistite acalculosa, os principais fatores de risco relacionados são queimaduras, cirurgias, traumas e nutrição parenteral (KIMURA Y, et al., 2013).

O objetivo deste estudo foi realizar uma revisão de literatura, englobando principalmente os aspectos de diagnóstico e tratamento da colecistite aguda, oferecendo assim subsídio para uma melhor decisão clínica frente a esta patologia.

\section{REVISÃO BIBLIOGRÁFICA}

A literatura mostra que ainda não há uma ferramenta clínica de alta precisão para o diagnóstico de colecistite aguda, já que o quadro do paciente e as alterações laboratoriais podem ser inespecíficos se analisados isoladamente, sendo fundamental a correlação com exames de imagem (ANSALONI L, et al., 2016). 
A avaliação por imagem deve ser realizada, inicialmente, pela ultrassonografia, método com bom custobenefício (ANSALONI L, et al., 2016; YOKOE M, et al., 2018). Em caso de indefinição diagnóstica, a colangiopancreatografia por ressonância magnética é a segunda escolha (YOKOE M, et al., 2018).

Conforme a diretriz de Tóquio de 2018, a colecistite aguda pode ser classificada em leve, moderada ou grave de acordo com o acometimento sistêmico e evolução do quadro (MIURA F, et al., 2013; YOKOE M, et al., 2018).

A terapêutica inicial é realizada com jejum, antibioticoterapia, controle hidroeletrolítico e analgesia (MIURA $\mathrm{F}$, et al., 2013), sendo posteriormente instituída a conduta cirúrgica (OKAMOTO K, et al., 2019) na qual a escolha para a maioria é a colecistectomia, salvo em pacientes instáveis e de alto risco, onde a drenagem percutânea deve ser considerada (MIURA F, et al., 2013).

Sabe-se que $5 \%$ a $15 \%$ dos pacientes podem apresentar coledocolitíase por um quadro de colecistite litiásica (ANSALONI L, et al., 2016). Assim, em casos de alto risco, como na presença de cálculos no ducto biliar durante avaliação ultrassonográfica, deve ser realizada investigação complementar com Colangiopancreatografia Retrógrada Endoscópica (CPRE) (ANSALONI L, et al., 2016).

\section{Diagnóstico}

Segundo a Ansolani L, et al. (2016), não existe nenhum achado clínico ou laboratorial com suficiente precisão diagnóstica para estabelecer ou excluir colecistite aguda, sendo que a combinação da anamnese, exame físico, exames laboratoriais e de imagem são necessários para apoiar com maior consistência o diagnóstico. No entanto, até o momento, não se conhece a melhor combinação de achados que possa melhorar a precisão diagnóstica.

Conforme o Guideline de Tóquio de 2013, mantidas no Guideline de 2018, para que haja um diagnóstico definitivo de colecistite, 3 critérios devem ser atendidos: A) sinal local de inflamação (sinal de Murphy ou dor, massa palpável ou sensibilidade em quadrante superior direito do abdome); B) sinal sistêmico de inflamação (febre, elevação da PCR, leucocitose); C) achados de imagem característicos de colecistite aguda. Um diagnóstico de suspeita pode ser feito quando da presença dos achados do item $A+B$, na ausência de achados de imagem (MIURA F, et al., 2013; OKAMOTO K, et al., 2019; YOKOE M, et al., 2018).

Sabe-se que a utilização dos critérios estabelecidos pelos Guidelines de Tóquio demonstra elevada especificidade $(96,9 \%)$ e sensibilidade $(91,2 \%)$ (MIURA F, et al., 2013; YOKOE M, et al., 2018). No entanto, a generalização destes resultados para a prática clínica pode ser problemática devido aos critérios de inclusão adotados no estudo japonês e aos achados de exame físico e alterações laboratoriais que tendem a ser mais discretas na população idosa, dificultando seu diagnóstico (ANSALONI L, et al., 2016; PISANO M, et al., 2017).

\section{Manifestação clínica e achados laboratoriais}

O sinal clínico mais comum é a dor abdominal em hipocôndrio direito ou epigástrio, desencadeada geralmente pela obstrução do colo da vesícula biliar ou do ducto cístico por um cálculo biliar, que apresenta sensibilidade variando de $56 \%$ a $93 \%$ e especificidade entre $0 \%$ e $96 \%$. Outros sinais/sintomas comumente referidos compreendem: náuseas (31-73\%), vômitos (60-83\%), febre superior a 38으 (cerca de 30\%), defesa muscular (30-58\%), massa palpável (14-25\%), sinal de Murphy (48-65\%) (MIURA F, et al., 2013; JAIN A, et al., 2017).

Em geral a clínica envolve uma dor em quadrante superior direito ou epigástrio, que pode irradiar para a região escapular direita, com duração superior a quatro horas, associada a náuseas ou vômitos, e normalmente em paciente com histórico de episódios álgicos semelhantes, desencadeada pela ingestão de alimentos gordurosos. Em alguns casos pode haver sinal de Murphy positivo (MIURA F, et al., 2013).

Icterícia também pode estar presente, que pode surgir decorrente de uma compressão extrínseca da via biliar devido a processo inflamatório, ou resultante de uma obstrução concomitante do colédoco. Pode existir 
em 5 a $15 \%$ dos pacientes com colecistite, ou ainda resultante da síndrome de Mirizzi, que ocorre em cerca de $1 \%$ dos casos de colecistite, e é caracterizada pela obstrução extrínseca da via biliar por um cálculo presente no ducto cístico ou no infundíbulo da vesícula biliar (ANSALONI L, et al., 2016).

Os achados laboratoriais mais comuns na colecistite são a leucocitose, aumento de PCR (normalmente acima de $3 \mathrm{mg} / \mathrm{dL}$ ), ligeiro aumento das enzimas séricas no sistema hepatobiliar-pancreático e bilirrubina (MIURA F, et al., 2013). Apesar de a literatura apontar para uma alteração laboratorial mais sutil em quadros de colecistite em pacientes idosos, isso ainda parece divergente (PISANO M, et al., 2017).

\section{Exames de imagem}

A ultrassonografia deve ser realizada sempre que houver suspeita da doença, devendo ser o primeiro exame a ser solicitado, tendo em vista seu fácil acesso, baixo custo, segurança, além de alta precisão para o diagnóstico de colelitíase, principal etiologia da colecistite (ANSALONI L, et al., 2016; MIURA F, et al., 2013; YOKOE M, et al., 2018; GONZÁLEZ-MUÑOZ Jl, et al., 2017).

Os achados de imagem característicos são: aumento da vesícula biliar, espessamento da parede da vesícula biliar (5 $\mathrm{mm}$ ou mais), cálculos na vesícula biliar (especialmente se impactado no colo da vesícula biliar ou ducto cístico), líquido pericolecístico e imagem sugestiva de gás na vesícula biliar (MIURA F, et al., 2013; YOKOE M, et al., 2018).

O sinal de Murphy ultrassonográfico (manifestação álgica quando se pressiona a vesícula biliar durante sua visualização por ultrassonografia) apresenta uma alta especificidade, podendo ser útil para o diagnóstico (MIURA F, et al., 2013). Ainda não há consenso quanto a utilização de Doppler (YOKOE M, et al., 2018).

A colangiopancreatografia por ressonância magnética é o método recomendado para os casos de indefinição diagnóstica na ultrassonografia (YOKOE M, et al., 2018). Os principais achados no diagnóstico de colecistite aguda são: espessamento da parede da vesícula biliar ( $\geq 4 \mathrm{~mm}$ ), aumento da vesícula biliar (eixo longo $\geq 8 \mathrm{~cm}$, eixo curto $\geq 4 \mathrm{~cm}$ ), cálculos biliares ou detritos retidos, acúmulo de líquido ao redor da vesícula biliar e sombras lineares no tecido adiposo ao redor da vesícula (YOKOE M, et al., 2018). O rendimento diagnóstico deste método é superior ao US, porém é pouco utilizado devido ao maior custo e menor disponibilidade. Destaca-se ainda sua superioridade no diagnóstico de colecistite gangrenosa em comparação a ultrassonografia (YOKOE M, et al., 2018).

Os principais achados na Tomografia Computadorizada (TC) são a distensão da vesícula biliar, espessamento da parede da vesícula, edema de subserosa, realce da mucosa, realce focal transitório do fígado adjacente a vesícula biliar, presença de líquido pericolecístico e abscesso pericolecístico. Na TC com contraste, observa-se um realce focal transitório no fígado adjacente a vesícula biliar na fase arterial, que desaparece na fase portal, sendo um importante sinal na colecistite leve em que é difícil avaliar a distensão da vesícula biliar (MIURA F, et al., 2013). Apresenta valor no diagnóstico da colecistite gangrenosa e enfisematosa (YOKOE M, et al., 2018).

A cintilografia hepatobiliar é realizada com a injeção de contraste venoso contendo marcadores radiossensíveis que são excretados por via biliar. A falha de enchimento da vesícula biliar após 60 minutos da administração do contraste assinala para obstrução da vesícula, sendo indicativo de colecistite aguda calculosa. Esse método apresenta uma especificidade e um grau de precisão maior que a ultrassonografia, mas é pouco disponível e de demorada realização. Dessa forma, a ultrassonografia é utilizada como primeiro exame devido a sua disponibilidade imediata, ausência de interferência por níveis elevados de bilirrubina sérica e ausência de radiação ionizante (ANSALONI L, et al., 2016; MIURA F, et al., 2013).

\section{Critérios de gravidade e diagnósticos diferenciais}

A colecistite aguda pode variar de uma condição leve, autolimitada, até uma doença fulminante e potencialmente fatal. A adoção de critérios de gravidade auxilia no manejo adequado dessa patologia. Segundo a diretriz de Tóquio 2013, mantidos na atualização de 2018, a colecistite é dividida em três 
categorias: leve (grau I), moderada (grau II), grave (grau III), que leva em consideração as repercussões sistêmicas e o grau de dificuldade de realização de uma colecistectomia (MIURA F, et al., 2013; YOKOE M, et al., 2018).

A colecistite aguda leve (grau I) é aquela que não apresenta achados de disfunção orgânica, possibilitando a realização de colecistectomia de forma segura e com baixo risco. A moderada (grau II) compreende os casos em que a inflamação local pode aumentar a dificuldade operatória durante a colecistectomia. Por fim, a grave (grau III) compreende os casos que apresentam disfunção orgânica. Estudos demonstram que essa classificação apresenta relação com mortalidade na admissão e prognóstico (YOKOE M, et al., 2018; JOSEPH B, et al., 2018). Os critérios da diretriz de Tóquio 2013/2018 são apresentados no Quadro 1 (MIURA F, et al., 2013; YOKOE M, et al., 2018).

Quadro 1 - Critérios de gravidade para colecistite aguda segundo diretriz de Tóquio 2013/2018.

\begin{tabular}{|c|l|}
\hline Gravidade & \multicolumn{1}{c|}{ Disfunção } \\
\hline Grau I (Leve) & $\begin{array}{l}\text { Não apresenta os critérios de colecistite grau II ou III. Pode também ser descrito } \\
\text { como colecistite aguda em pacientes sem disfunção orgânica, pequenas alterações } \\
\text { inflamatórias da vesícula biliar, tornando a colecistectomia um procedimento } \\
\text { seguro e de baixo risco. }\end{array}$ \\
\hline \multirow{3}{*}{ Grau II (Moderada) } & $\begin{array}{l}\text { Leucocitose }>18.000 / \mathrm{mm}^{3} ; \\
\text { Massa palpável, sensível, em quadrante superior direito do abdome; } \\
\text { Tempo de evolução superior a } 72 \text { horas; } \\
\text { Sinais de inflamação local sugestivo de: colecistite gangrenosa, abscesso } \\
\text { pericolecístico, peritonite biliar, colecistite enfisematosa. }\end{array}$ \\
\hline Grau III (Grave) & $\begin{array}{l}\text { Disfunção cardiovascular: hipotensão que requer tratamento com dopamina em } \\
\text { doses iguais ou superiores a } 5 \text { mcg/kg/min, ou qualquer dose de norepinefrina; } \\
\text { Disfunção neurológica: diminuição do nível de consciência; } \\
\text { Disfunção respiratória: } \mathrm{PaO} / \mathrm{FiO} 2<300 ; \\
\text { Disfunção hepática: INR }>1,5 ; \\
\text { Disfunção renal, oligúria, creatinina }>2.0 \mathrm{mg} / \mathrm{dL} \\
\text { Disfunção hematológica: contagem de plaquetas }<100.000 / \mathrm{mm}^{3} .\end{array}$ \\
\hline
\end{tabular}

Fonte: Junior ES, et al., 2021; dados extraídos de Miura F, et al., 2013 e Yokoe M, et al., 2018.

Outras patologias também evoluem com dor em hipocôndrio direito, por isso a importância de se realizar o diagnóstico diferencial. A Colangite Esclerosante Primária (CEP) é uma doença colestática rara imunomediada, em que ocorre fibrose da árvore biliar por desordem imunológica, agentes tóxicos, injúria ao ducto biliar e alteração no fluxo hepatobiliar (SANTOS PAM, 2016). Há associação com a doença inflamatória intestinal, podendo progredir para a formação de cirrose, hipertensão portal e insuficiência hepática (CARVALHO-FILHO RJ, et al., 2019). Na fase tardia, cursa com prurido, dor em hipocôndrio direito e febre com calafrio, e pode haver elevação das aminotransferases. O diagnóstico definitivo é feito em pacientes com clínica de colestase e um exame de imagem - colangioressonância magnética ou colangiografia retrógrada endoscópica, em que será visível alteração nos ductos biliares (KE L, et al., 2014).

A pancreatite aguda também deve ser descartada em caso de dor na topografia superior do abdômen com náuseas e vômitos. As etiologias mais comuns são a de origem biliar e alcóolica. O diagnóstico inicial é realizado pela clínica típica, elevação 3 vezes o valor da normalidade da amilase e lipase e por alterações visíveis na tomografia computadorizada (HATOUM US, et al., 2015). A forma leve da doença corresponde a aproximadamente $85 \%$ dos casos, e geralmente cursa com alta hospitalar em até 7 dias do início da doença (LACERDA PS, et al., 2014). 
Outro diagnóstico diferencial da colecistite aguda é a Síndrome de Mirizzi, uma rara doença colestática extra-hepática em portadores de colelitíase crônica, mais comum em mulheres. Ocorre a obstrução do ducto hepático ou colédoco devido a compressão dos cálculos biliares no ducto cístico ou no infundíbulo vesicular. Como complicação, pode haver uma fístula colecistobiliar ou colecistoentérica, e nesta o paciente poderá desenvolver uma obstrução intestinal conhecida como íleo biliar. Os exames laboratoriais e sintomas são poucos específicos, e envolvem dor abdominal, icterícia e colangite, mas pode estar associado a náuseas, vômitos, colúria, prurido e hepatomegalia. Embora só ocorra em até $4 \%$ dos pacientes com colelitíase, o diagnóstico diferencial é importante devido ao risco de lesar o ducto biliar durante a colecistectomia (MOU D, et al., 2019).

\section{Tratamento da colecistite}

As medidas a serem adotadas incluem jejum, antibioticoterapia, manutenção de um adequado balanço ácido base e hidroeletrolítico, analgesia, antibioticoterapia e monitoramento continuado. Essas medidas iniciais oferecem um cenário favorável para instituição do tratamento intervencionista, que engloba a cirurgia e a drenagem da via biliar (MIURA F, et al., 2013). Não existe ainda outra possibilidade com resultados documentados para o tratamento de colecistite, portanto tentativas de dissolução dos cálculos ou litotripsia extracorpórea por ondas de choque não possuem indicação no momento (ANSALONI L, et al., 2016).

Entre os analgésicos, os AINES mostraram-se úteis em prevenir a instalação da colecistite aguda em casos de dores decorrentes de colelitíase. Entretanto, uma vez instalado o processo, tais drogas não alteram a evolução da doença (MIURA F, et al., 2013).

A antibioticoterapia deve ser sugerida como tratamento de suporte, pois apesar da colecistite ser um processo inflamatório no início, a estase biliar contínua pode desencadear uma infecção secundária da vesícula e possibilitar uma sepse (ANSALONI L, et al., 2016). A escolha do antibiótico envolve o conhecimento dos patógenos mais comuns - aeróbios gram negativos (E. coli e K. pneumoniae) e anaeróbios (Bacteroides fragilis) (CAMPANILLE FC, et al., 2014).

A cultura e antibiograma da bile é útil para os casos complicados e de alto risco de resistência microbiana, o que permite personalizar o tratamento antimicrobiano garantindo maior eficácia, embora a positividade da bile ou vesícula biliar variem de 29 a 54\%. Os casos não complicados em que o foco de infecção é controlado após a colecistectomia não necessitam de antibioticoterapia (ANSALONI L, et al., 2016).

De maneira geral, o tratamento de primeira linha depois de instituído as medidas iniciais é a colecistectomia precoce ou urgente, sendo a via laparoscópica a preferida (OKAMOTO K, et al., 2019; COCCOLINI F, et al., 2015). A instituição do tratamento laparoscópico precoce deve ser preferida à intervenção tardia, uma vez que demonstrou melhores resultados, pois não se observaram diferenças significativas relativas nas taxas de conversão para cirurgia aberta, tempo de operação e taxas de complicações globais. Além disso, a via laparoscópica oferece menor tempo de internação e, com isso, maior economia (CAMPANILLE FC, et al., 2014; KOTI RS, et al., 2015; MENAHEM B, et al., 2015; CHARLSON ME, et al., 1987). Algumas situações que se recomenda a conversão para a técnica são: inflamação local intensa, aderências, hemorragias no triângulo de Calot ou suspeita de lesão biliar (ANSALONI L, et al., 2016).

Quanto ao tempo desde o diagnóstico, o protocolo de Tóquio 2018, propõem que desde que o paciente tenha condições de resistir à cirurgia, a colecistectomia videolaparoscopia precoce é a escolha terapêutica, inclusive para os casos de diagnóstico tardio. Essa abordagem está relacionada com menor tempo de internação e custos hospitalares (OKAMOTO K, et al., 2019). O Guideline de Tóquio 2018, sugere a instituição do tratamento de acordo com o grau da colecistite, associado a fatores de risco, utilizando como referência alguns fatores preditivos já documentados, o Charlson Comorbidity Index $(\mathrm{CCl})$ e o American Society of Anesthesiology Physical Status (ASA-PS), como apresentado no Quadro 2 (CHARLSON ME, et al., 1987; OKAMOTO K, et al., 2019). O Quadro 3 e o Quadro 4 representam os critérios do CCI e ASA-PS respectivamente (CHARLSON ME, et al., 1987; AMERICAN SOCIETY OF ANESTHESIOLOGY PHYSICAL STATUS CLASSIFICATION SYSTEM, 2014). 
Quadro 2 - Tratamento da colecistite aguda de acordo com a gravidade segundo o Guideline de Tóquio 2018.

\begin{tabular}{|c|l|}
\hline Gravidade & \multicolumn{1}{c|}{ Tratamento } \\
\hline Grau I (Leve) & $\begin{array}{l}\text { Colecistectomia laparoscópica é o tratamento de primeira linha, podendo ser } \\
\text { instituído tratamento clínico e observação para aqueles com risco cirúrgico muito } \\
\text { alto, utilizando-se como referência o CCI e o ASA-PS }\end{array}$ \\
\hline Grau II (Moderada) & $\begin{array}{l}\text { Colecistectomia precoce caso os escores CCI e ASA-PS indicarem que o paciente } \\
\text { possa resistir a cirurgia, sendo ainda recomendada a realização de cirurgia em } \\
\text { centro avançado, com cirurgião experiente. Recomenda-se redobrar os cuidados } \\
\text { para evitar lesões ao decorrer da cirurgia, podendo-se recorrer a colecistectomia } \\
\text { subtotal ou colecistectomia aberta a depender das condições. Caso o paciente não } \\
\text { possua condições clínicas para o procedimento cirúrgico, deve-se considerar o } \\
\text { tratamento conservador e drenagem biliar. }\end{array}$ \\
\hline Grau III (Grave) & $\begin{array}{l}\text { Após oferta do suporte adequado às disfunções orgânicas (suporte ventilatório, } \\
\text { estabilização hemodinâmica e outros) e antibioticoterapia, deve-se avaliar a } \\
\text { resposta ao tratamento. Caso seja positiva, e os escores CCI e ASA-PS sejam } \\
\text { favoráveis, na ausência de disfunção neurológica, respiratória e icterícia } \\
\text { (Bilirrubina total <2 mg/dL), pode-se realizar a colecistectomia videolaparoscópica } \\
\text { precoce, em centro de referência, com suporte intensivo e cirurgião especialista. } \\
\text { Caso o paciente não apresente condições clínicas para a cirurgia, deve-se instituir } \\
\text { tratamento conservador abrangente, considerando drenagem biliar precoce, } \\
\text { reservando colecistectomia após a melhora clínica do paciente. }\end{array}$ \\
\hline
\end{tabular}

Nota: 1- Colecistite aguda grau I e II com CCl > 6 e ASA-PS > 3, e grau III com CCl > 4 e ASA-PS > 3 estão associados a um risco maior de mortalidade, sendo indicado tratamento clínico e colecistectomia laparoscópica assim que o paciente apresentar condições. 2- Para os pacientes com colecistite grau III, são apontados como fatores preditivos negativos a disfunção neurológica, disfunção respiratória e coexistência de icterícia (bilirrubina total $\geq 2 \mathrm{mg} / \mathrm{dL}$ ), sendo indicado tratamento clínico e colecistectomia laparoscópica assim que o paciente apresentar condições. 3- Dentre as disfunções que podem estar presentes no grau III, as disfunções cardiovasculares e renais são listadas como disfunções orgânicas responsivas a medidas iniciais de tratamento, não sendo impeditivas absolutas para realização de colecistectomia videolaparoscópica precoce. Fonte: Junior ES, et al., 2021; dados extraídos de Okamoto, et al., 2019.

Quadro 3 - Charlson Comorbidity Index.

\begin{tabular}{|c|l|}
\hline Peso atribuído & \multicolumn{1}{c|}{ Condição } \\
\hline & Infarto do miocárdio \\
& Insuficiência cardíaca congestiva \\
& Doença vascular periférica \\
Doença cerebrovascular & Demência \\
Doenças pulmonar crônica \\
Doença do tecido conjuntivo \\
Doença ulcerosa péptica \\
& Diabete mellitus sem complicações \\
\hline & Hemiplegia \\
& Doença renal crônica moderada ou grave \\
& Diabetes mellitus, com lesão de órgão alvo \\
& Qualquer tumor sólido \\
& Leucemia \\
& Linfoma \\
\hline 3 & Doença hepática moderada ou grave. \\
\hline 1 & Cada década de idade acima de 40 anos. \\
\hline 6 & Tumor sólido metastático \\
& Síndrome da Imunodeficiência Adquirida (AIDS). \\
\hline
\end{tabular}

Fonte: Junior ES, et al., 2021; dados extraídos de Charlson ME, et al., 1987. 
Quadro 4 - Classificação ASA-PS.

\begin{tabular}{|c|c|c|}
\hline $\begin{array}{l}\text { Classificação } \\
\text { ASA-PS }\end{array}$ & Definição & Exemplos \\
\hline ASA I & $\begin{array}{l}\text { Paciente } \\
\text { saudável }\end{array}$ & Saudável, não tabagista, sem ingesta alcoólica ou ingesta mínima. \\
\hline ASA II & $\begin{array}{l}\text { Paciente com } \\
\text { doença } \\
\text { sistêmica leve }\end{array}$ & $\begin{array}{l}\text { Doenças leves, sem limitações funcionais substanciais. Os exemplos } \\
\text { incluem (mas não se limitam a): tabagista atual, consumo social de } \\
\text { álcool, gravidez, obesidade (IMC < 40), diabetes / hipertensão bem } \\
\text { controlada, doença pulmonar leve; }\end{array}$ \\
\hline ASA III & $\begin{array}{l}\text { Paciente com } \\
\text { doença } \\
\text { sistêmica grave }\end{array}$ & $\begin{array}{l}\text { Doença com limitação funcional: uma ou mais doenças moderadas a } \\
\text { graves. Os exemplos incluem (mas não se limitam a): Diabetes } \\
\text { Mellitus (DM) ou Hipertensão Arterial Sistêmica (HAS) mal controlada, } \\
\text { Doença Pulmonar Obstrutiva Crônica (DPOC), obesidade mórbida } \\
\text { (IMC > 40), hepatite ativa, dependência ou abuso de álcool, } \\
\text { marcapasso implantado, redução da fração de ejeção, doença renal } \\
\text { crônica dialítica, história de infarto agudo do miocárdio (IAM) ou } \\
\text { Acidente Vascular Encefálico (AVE) há mais de } 3 \text { meses. }\end{array}$ \\
\hline ASA IV & $\begin{array}{l}\text { Doença } \\
\text { sistêmica grave, } \\
\text { com ameaça } \\
\text { constante a vida }\end{array}$ & $\begin{array}{l}\text { Os exemplos incluem (mas não se limitam a): história recente (a } \\
\text { menos de } 3 \text { meses) de IAM, AVE, AIT, redução severa da fração de } \\
\text { ejeção, sepse, CIVD, doença respiratória aguda, doença renal em } \\
\text { estágio terminal, não sendo submetidas regularmente a programa de } \\
\text { diálise. }\end{array}$ \\
\hline ASA V & $\begin{array}{l}\text { Paciente } \\
\text { moribundo, sem } \\
\text { expectativa de } \\
\text { sobrevivência } \\
\text { sem cirurgia }\end{array}$ & $\begin{array}{l}\text { Os exemplos incluem (mas não se limitam a): disfunção de múltiplos } \\
\text { órgãos / sistemas, ruptura de aneurisma torácico ou abdominal, } \\
\text { trauma extenso, hemorragia intracraniana com efeito massa, isquemia } \\
\text { intestinal decorrente de patologia cardíaca significativa }\end{array}$ \\
\hline ASA VI & $\begin{array}{l}\text { Paciente com } \\
\text { morte cerebral }\end{array}$ & $\begin{array}{l}\text { Paciente com morte cerebral cujos órgãos serão removidos para fins } \\
\text { de doação }\end{array}$ \\
\hline
\end{tabular}

Legenda: AIT: Ataque Isquêmico Transitório; AVE: Acidente Vascular Encefálico; CIVD: Coagulação Intravascular Disseminada; DC: Doença coronariana; DM: Diabetes mellitus; DPOC: Doença pulmonar obstrutiva crônica; HAS: Hipertensão arterial sistêmica; IMC: índice de massa corporal.

Fonte: Junior ES, et al., 2021; dados extraídos de ASA, 2014.

Para pacientes de alto risco em que as condições momentâneas não favorecem uma colecistectomia, está indicada a drenagem percutânea da vesícula biliar ou a drenagem endoscópica, reservando a colecistectomia após a estabilização clínica (MIURA F, et al., 2013).

A via de drenagem endoscópica parece ser eficaz e mais segura que a drenagem percutânea, merecendo novos estudos para sua consolidação (MIURA F, et al., 2013; ROSALES-VELDERRAIN A E ALKHOURY F, 2017). O objetivo da drenagem é converter uma colecistite séptica em uma condição não séptica, reduzindo assim os sintomas e a resposta inflamatória (MIURA F, et al., 2013; VISTE A, et al., 2015).

Dentre as técnicas disponíveis, a drenagem da vesícula biliar trans-hepática percutânea é a preferida devido a facilidade e custos reduzidos (ANSALONI L, et al., 2016). Ainda não há consenso sobre o momento ideal para realização da colecistectomia após a drenagem biliar (OKAMOTO K, et al., 2018; COCCOLINI F, et al., 2015).

Em se tratando de idosos, a colecistectomia laparoscópica precoce é o tratamento de escolha caso as condições clínicas permitam. Caso haja contraindicações ao procedimento, a drenagem biliar deve ser instituída, de preferência pela via percutânea trans-hepática, deixando a colecistectomia para posteriormente (PISANO M, et al., 2017). 
Quanto maior a gravidade do episódio de colecistite, maiores são as dificuldades técnicas operatórias e chances de complicações, como lesão de via biliar (COCCOLINI F, et al., 2015). São fatores de risco que predizem uma operação difícil: sintomas com duração maior que 72 horas, contagem de leucócitos superior a 18.000 / mm3, vesícula biliar palpável e colecistite gangrenosa (KARLSEN TH, et al., 2017). Nos casos em que o processo inflamatório seja intenso, dificultando a dissecção das estruturas, pode-se realizar: colangiografia intraoperatória (para definir a anatomia biliar), colecistectomia subtotal (deixando a parede posterior da vesícula biliar no fígado) e conversão para cirurgia aberta, quando o julgamento crítico determinar que a via aberta facilitará a dissecção (COCCOLINI F, et al., 2015).

\section{Exploração das vias biliares na colecistite}

Cerca de 5 a 15\% dos casos de colecistite podem apresentar coledocolitíase durante um episódio de colecistite litiásica. (ANSALONI L, et al., 2016). Devido a essa baixa incidência e ao maior tempo de intervenção, exames complementares para investigação de coledocolitíase devem ser utilizados em casos selecionados, com alta probabilidade de existência de cálculos em vias biliares (CAMPANILLE FC, et al., 2014).

A ultrassonografia é um exame pouco invasivo e amplamente disponível. Pode ser utilizado na investigação inicial de coledocolitíase (YOKOE M, et al., 2018). Em caso de visualização de cálculos no colédoco, a investigação pode prosseguir com uso de colangiopancreatografia retrógrada endoscópica pré, intra ou pós-operatória, ou colangiografia intraoperatória, ou ultrassonografia laparoscópica a depender da experiência e disponibilidade local. Pacientes com quadros de colecistite de médio risco devem ser investigados a partir de colangiorressonância por ressonância magnética, ultrassonografia endoscópica, ultrassonografia laparoscópica intraoperatória ou colangiografia intraoperatória, evitando assim a CPRE. Para os indivíduos de baixo risco, a investigação para coledocolitíase não é encorajada (ANSALONI L, et al., 2016).

\section{CONSIDERAÇÕES FINAIS}

O diagnóstico da colecistite aguda consiste na combinação da anamnese, exame físico, exames laboratoriais e de imagens. Entretanto, até o momento, não se conhece a melhor combinação de achados que possa ampliar a precisão diagnóstica. A ultrassonografia deve ser realizada sempre que houver suspeita da doença, devendo ser o primeiro exame a ser solicitado. Pacientes com alta suspeição de coledocolitíase podem se beneficiar de exames adicionais para identificação de cálculos em vias biliares, como a colangiopancreatografia retrógrada endoscópica, colangiografia intraoperatória ou ultrassonografia laparoscópica. Quanto ao tratamento, a conduta inicial inclui estabelecimento de jejum, antibioticoterapia e disponibilização de condições de suporte para a doença, oferecendo um cenário favorável para instituição do tratamento intervencionista, que engloba a cirurgia e a drenagem da via biliar.

\section{REFERÊNCIAS}

1. ABADÍA-BARNÓ P, et al. Gallbladder volvulus: Diagnostic and surgical challenges. Cirugia y cirujanos (English Ed), 2017; 85: 89-92.

2. ANSALONI L, et al. 2016 WSES guidelines on acute calculous cholecystitis. World Journal of Emergency Surgery [Internet], 2016; 11(1): 1-23.

3. AMERICAN SOCIETY OF ANESTHESIOLOGY PHYSICAL STATUS CLASSIFICATION SYSTEM (ASA-OS). 2014. In: ASA HQ. Schaumburg: American Society of Anesthesiology. Disponível em: <https://www.asahq.org/standardsand-guidelines/asa-physical-status-classification-system>. Acesso em: 10 jan. 2021.

4. CARVALHO FILHO RJ, SILVA AEB. Colangite esclerosante primária. In Bittencourt PL, Couto CA. Manual de conduta em doenças colestáticas e autoimunes do fígado. [Internet], 2019; $1: 28-33$.

5. CAMPANILLE FC, et al. Acute cholecystitis: WSES position statement. World Journal of Emergency Surgery, 2014; 9(1): 1-6.

6. CHARLSON ME, et al. A new method of classifying prognostic comorbidity in longitudinal studies: Development and validation. Journal of Chronic Diseases, 1987; 40(5): 373-83.

7. COCCOLINI F, et al. Open versus laparoscopic cholecystectomy in acute cholecystitis. Systematic review and metaanalysis. International Journal of Surgery [Internet]. 2015; 18: 196-204.

8. GOMES CA, et al. Gangrenous cholecystitis in male patients: A study of prevalence and predictive risk factors. Annals of Hepatobiliary Pancreatic Surgery. 2019; 23(1): 34-40. 
9. GONZÁLEZ-MUÑOZ Jl, et al. Risk-adjusted treatment selection and outcome of patients with acute cholecystitis. Langenbeck's Archives of Surgery [Internet], 2017; 402(4): 607-614.

10. HATOUM US, et al. Escores prognósticos em pancreatite aguda: revisão de literatura. Anais eletrônico: IX EPCCEncontro Internacional de Produção Cientifica UniCesumar, 2015; 9: 4-8.

11. ITOI T. New era in acute cholecystitis treatment: encouraging results for interventional endoscopists. Gastrointestinal Endoscopic [Internet], 2017; 85(1): 88-89.

12. JAIN A, et al. History, Physical Examination, Laboratory Testing, and Emergency Department Ultrasonography for the Diagnosis of Acute Cholecystitis. Academic Emergency Medicine, 2017; 24: 281-297.

13. JOSEPH B, et al. Evaluating the Relevance of the 2013 Tokyo Guidelines for the Diagnosis and Management of Cholecystitis. Journal of the American College of Surgeons [Internet], 2018; 227(1): 38-43.

14. KARLSEN TH, et al. Primary sclerosing cholangitis - a comprehensive review. Journal of Hepatology [Internet], 2017 ; 67(6): 1298-323.

15. KE L, et al. Predictors of critical acute pancreatitis: a prospective cohort study. Medicine, 2014; 93(21): 108.

16. KIMURA Y, et al. TG13 current terminology, etiology, and epidemiology of acute cholangitis and cholecystitis. Journal of Hepato-Biliary-Pancreatic Sciences. 2013; 20(1): 8-23.

17. KOTI RS, et al. Surgical management of acute cholecystitis. Langenbeck's Archives of Surgery, 2015; 400(4): 403-19.

18. LACERDA OS, et al. Síndrome de Mirizzi: um grande desafio cirúrgico. ABCD - Arquivos Brasileiros de Cirurgia Digestiva, 2014; 27(3): 226-227.

19. MENAHEM B, et al. Delayed laparoscopic cholecystectomy increases the total hospital stay compared to an early laparoscopic cholecystectomy after acute cholecystitis: an updated meta-analysis of randomized controlled trials. HPB: the official journal of the International Hepato Pancreato Biliary Association, 2015; 1: 857-862.

20. MIURA F, et al. TG13 flowchart for the management of acute cholangitis and cholecystitis. Journal of Hepato-BiliaryPancreatic Sciences, 2013; 20(1): 47-54.

21. MOU D, et al. Advances in the management of acute cholecystitis. Annals of Gastroenterological Surgery, 2019; 3(3): 247-253.

22. OKAMOTO K, et al. Tokyo Guidelines 2018: flowchart for the management of acute cholecystitis. Journal of HepatoBiliary-Pancreatic Sciences, 2019; 26(11): 534.

23. PISANO M, et al. 2017 WSES and SICG guidelines on acute calcolous cholecystitis in elderly population. World Journal of Emergency Surgery, 2019;14(1): 1-16.

24. ROSALES-VELDERRAIN A, ALKHOURY F. Single-Port Robotic Cholecystectomy in Pediatric Patients: Single Institution Experience. Journal of laparoendoscopic \& advanced surgical techniques, 2017; 27(4): 434-437.

25. SANTOS PAM. Colangite esclerosante primária: um estudo retrospectivo no CHUC. Dissertação (Mestrado integrado em Medicina) - Faculdade de Medicina da Universidade de Coimbra, Coimbra, 2016; 47p

26. SCHULD J, GLANEMANN M. Acute Cholecystitis. Viszeral medizin, 2015; 31(3): 163-165.

27. VISTE A, et al. Percutaneous cholecystostomy in acute cholecystitis; a retrospective analysis of a large series of 104 patients. BMC Surgery, 2015; 1: 15-17.

28. YOKOE M, et al. Tokyo Guidelines 2018: diagnostic criteria and severity grading of acute cholecystitis (with videos). Journal of Hepato-Biliary-Pancreatic Sciences, 2018; 25(1): 41-54. 